\title{
Blue quantum electroabsorption modulators based on reversed quantum confined Stark effect with blueshift
}

\author{
Emre Sari, Sedat Nizamoglu, Tuncay Ozel, and Hilmi Volkan Demir ${ }^{\mathrm{a})}$ \\ Deparment of Physics, Bilkent University, Bilkent, Ankara 06800, Turkey; Department of Electrical and \\ Electronics Engineering, Bilkent University, Bilkent, Ankara 06800, Turkey; and Nanotechnology \\ Research Center, Bilkent University, Bilkent, Ankara 06800 Turkey
}

(Received 18 October 2006; accepted 22 November 2006; published online 2 January 2007)

\begin{abstract}
The authors present the design, growth, fabrication, experimental characterization, and theoretical analysis of blue quantum electroabsorption modulators that incorporate $\sim 5 \mathrm{~nm}$ thick $\mathrm{In}_{0.35} \mathrm{Ga}_{0.65} \mathrm{~N} / \mathrm{GaN}$ quantum structures for operation between 420 and $430 \mathrm{~nm}$. Growing on polar $c$ plane on sapphire, they obtain quantum structures with zigzag potential profile due to alternating polarization fields and demonstrate that their optical absorption blueshifts with applied electric field, unlike the redshift of conventional quantum confined Stark effect. In InGaN/GaN quantum structures, they report the largest absorption change of $6000 \mathrm{~cm}^{-1}$ for $6 \mathrm{~V}$ bias swing around $424 \mathrm{~nm}$, holding promise for blue optical clock generation and injection directly into silicon chips. (C) 2007 American Institute of Physics. [DOI: 10.1063/1.2424642]
\end{abstract}

Today silicon microelectronics is limited in operating speed: the electrical interconnects suffer from the $R C$ limitation; scaling does not mitigate this problem. ${ }^{1}$ This leads to a bottleneck in electrical clocking. Optical clocking is proposed as a remedy. ${ }^{2}$ Optical clock distribution is implemented commonly in the near infrared (IR) spectral region where optoelecronic devices are readily available. ${ }^{3,4}$ However, silicon photodetectors, for example, those fabricated in standard complementary metal-oxide-semiconductor (CMOS) process, unfavorably exhibit long absorption length and thus a diffusion tail problem in the near IR. This limits the operating speed of $\mathrm{Si}$ CMOS photodetectors to $1 \mathrm{Gbits} / \mathrm{s}^{5}{ }^{5}$ To circumvent this problem, one approach is to utilize high-speed III-V photodetectors hybrid integrated on $\mathrm{Si}$ chips. This, however, introduces difficulties related to post-CMOS fabrication. On the other hand, unlike in the IR, optical clock injection directly to $\mathrm{Si}$ is possible in the blue, where Si features a very short absorption length $(\sim 100 \mathrm{~nm}$ at $400 \mathrm{~nm}$ ) and thus lacks the diffusion tail. ${ }^{6}$ However, there exists no chip-scale device to modulate optical clock signal in the blue at high speeds to date. In this letter, we propose blue InGaN/GaN based quantum electroabsorption modulators for a possible chip-scale solution in optical clock modulation in the blue. Here we present the design, epitaxial growth, fabrication, experimental characterization, and theoretical analysis of our quantum electroabsorption modulators that incorporate $\sim 5 \mathrm{~nm}$ thick $\operatorname{In}_{0.35} \mathrm{Ga}_{0.65} \mathrm{~N} / \mathrm{GaN}$ quantum structures in a $p$ - $i$ - $n$ diode architecture for operation in the blue spectral range, as shown in Fig. 1.

We grow these quantum structures on the polar $c$ plane of $\mathrm{GaN}$ on sapphire in metal organic chemical vapor deposition (MOCVD) and obtain a zigzag potential profile due to high polarization fields with alternating directions in their heterostructures. We study the electroabsorption behavior of these quantum zigzag structures for proof-of-concept demonstration of their use in blue modulation. In the blue range, using InGaN/GaN quantum structures, we experimentally demonstrate the largest optical absorption change of $6000 \mathrm{~cm}^{-1}$ with the application of a reverse bias of $-6 \mathrm{~V}$

${ }^{a)}$ FAX: [+90](312)290-1015; electronic mail: volkan@bilkent.edu.tr across our diodes (corresponding to $50 \mathrm{~cm}^{-1} / 1 \mathrm{~V} / \mu \mathrm{m}$ field swing) at $424 \mathrm{~nm}$ in the highly transmissive region below the band edge. This implies that a modulation contrast ratio of $>10 \mathrm{~dB}$ is possible with an optical interaction length $\sim 100 \mu \mathrm{m}$ and a field swing of $2 \mathrm{~V} / \mu \mathrm{m}$ in principle. Opposite to the typical redshift of the traditional quantum confined Stark effect (QCSE), we demonstrate in these zigzag structures that the band edge shifts to shorter wavelengths (blueshifts) with the increasing applied reverse field, as plotted in Fig. 1. Our theoretical analysis shows that this reversed behavior of the quantum confined Stark effect stems from high alternating polarization fields.

The conventional quantum structures in nonpolar III-V semiconductors form a steplike potential profile. In such square quantum well structures, we observe quantum confined Stark effect, ${ }^{7}$ which brings the electron and hole energy states closer in energy when an external electric field is applied (in either direction). Thus, the traditional QCSE redshifts the optical absorption edge with respect to the no-field case (where the energy bands are flat). However, III-N based quantum structures form a zigzag potential profile due to the

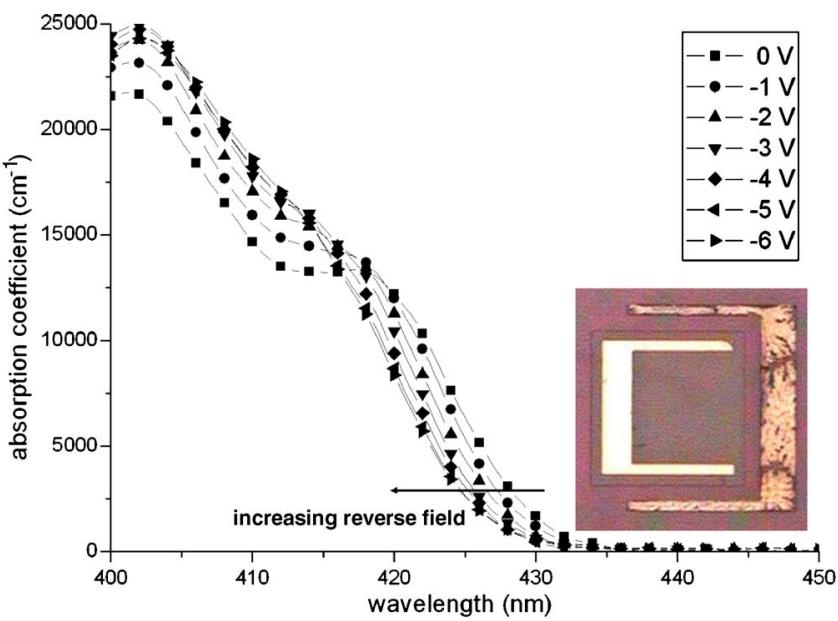

FIG. 1. (Color online) Optical absorption spectra measured from our InGaN/GaN based quantum electroabsorption modulator parametrized with the applied reverse bias at room temperature, along with a top-view micrograph of one of our fabricated devices. 

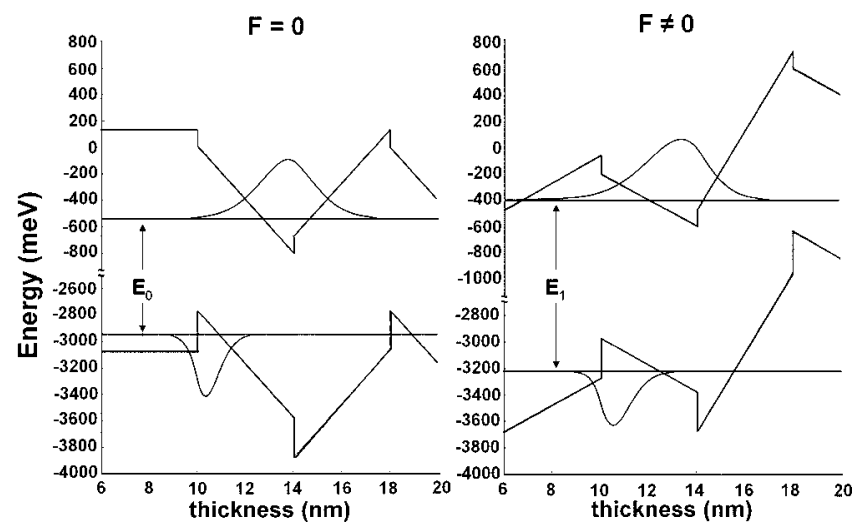

FIG. 2. Electron and hole energy states (a) for the nonbiased case $(F=0)$ and $(b)$ for the biased case $(F \neq 0)$, simulated by the transfer matrix method. Here $E_{1}>E_{0}$, which implies $\lambda_{1}<\lambda_{0}$ for the corresponding absorption band edges.

high alternating polarization fields. ${ }^{8}$ For such quantum zigzag structures, our quantum mechanical simulations based on transfer matrix method demonstrate that the band edge blueshifts as presented in Fig. 2, verifying our experimental observation in Fig. 1, when an external electric field is applied in the opposite direction of the polarization field seen in the wells. Comparing Fig. 2(a) for the zero-field case $(F=0)$ and Fig. 2(b) for the non-zero-field case $(F \neq 0$ in the opposite direction of the well polarization), we observe that $E_{1}>E_{0}$ and thus $\lambda_{1}<\lambda_{0}$, where $E_{0,1}$ are the energy separations between the first electron and hole states and $\lambda_{0,1}$ are the corresponding absorption band edges in the respective (a) $F$ $=0$ and (b) $F \neq 0$ cases. Here we understand that this reversed QCSE arises because the electric field applied in the opposite direction of the well polarization compensates for the polarization and brings the quantum zigzag structure closer to the flat energy band condition in the wells. On the other hand, when the external field is applied in the direction of the well polarization field, we observe the typical QCSE with a redshift, rather than the reversed QCSE with a blueshift.

The epitaxial design of our modulators is based on a $p-i-n$ structure that houses InGaN/GaN quantum structures in its intrinsic region. We grow our epitaxial wafers on $c$-plane double side polished sapphire substrates using our AIXTRON RF200/4 RF-S MOCVD system located at Bilkent University Nanotechnology Research Center. Our epitaxial growth starts with a $14 \mathrm{~nm}$ thick low-temperautre $\mathrm{GaN}$ nucleation layer and a $200 \mathrm{~nm}$ thick high-temperature GaN buffer layer for a high crystal quality and then follows a $690 \mathrm{~nm}$ thick Si doped $\mathrm{GaN}$ layer for $n$-type contact. Subsequently we grow our active region that consists of five $\sim 5 \mathrm{~nm}$ thick $\mathrm{In}_{0.35} \mathrm{Ga}_{0.65} \mathrm{~N}$ well and $\sim 5 \mathrm{~nm}$ thick $\mathrm{GaN}$ barrier structures at a constant temperature of $682{ }^{\circ} \mathrm{C}$. We finalize our growth with $\mathrm{Mg}$ doped, $50 \mathrm{~nm}$ thick $\mathrm{Al}_{0.1} \mathrm{Ga}_{0.9} \mathrm{~N}$ and $120 \mathrm{~nm}$ thick GaN layers-both $p$ type, with the latter serving as the $p$-contact layer. In our MOCVD epigrowth, we use TEGa (for quantum structures), TMGa (for bulk layers), TMIn, TMAl, and $\mathrm{NH}_{3}$ as the precursors.

We start the device fabrication with the dehydrogenation of $\mathrm{Mg}$ dopants by annealing our epitaxial wafers at $750{ }^{\circ} \mathrm{C}$ for $15 \mathrm{~min}$ in $\mathrm{N}_{2}$ ambient atmosphere. We use standard lithography for reactive ion etching of device mesas and subsequent metallization steps. We evaporate Ni:Au and Ti:Al layers (both $10 / 100 \mathrm{~nm}$ thick) for $p$ and $n$ contacts, respec-

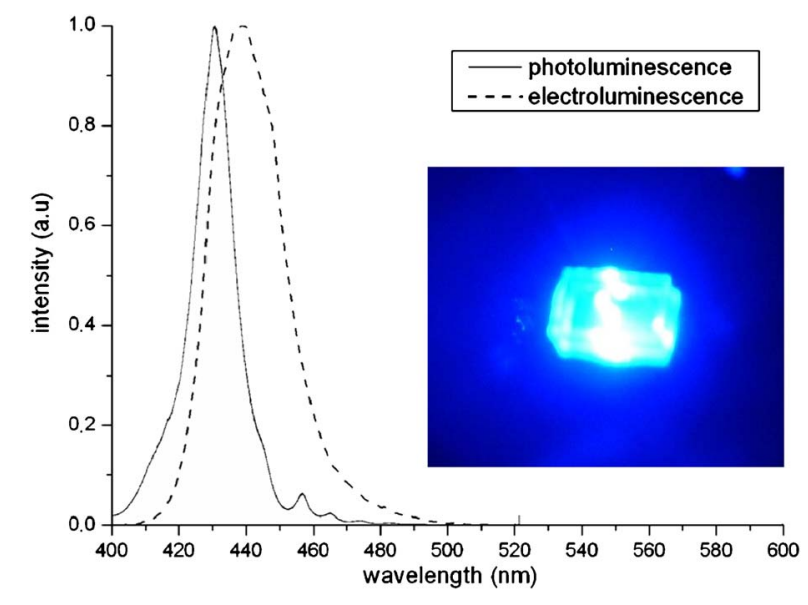

FIG. 3. (Color online) Photoluminesence and electroluminesence spectra of our InGaN/GaN based quantum electroabsorption modulator, along with a micrograph of the emitted blue light when the diode is forward biased.

tively. We finally apply rapid thermal annealing at $650{ }^{\circ} \mathrm{C}$ for $1 \mathrm{~min}$. Our fabricated devices have mesa sizes varying from $10 \times 10$ to $300 \times 300 \mu \mathrm{m}^{2}$, with open optical windows to couple light into and out of the mesa in surface-normal operation.

We perform photoluminescence (PL) characterization at room temperature using a $\mathrm{He}-\mathrm{Cd}$ laser at an excitation wavelength of $325 \mathrm{~nm}$. We observe the PL peak at $430 \mathrm{~nm}$ with a full width at half maximum (FWHM) of $14 \mathrm{~nm}$, as shown in Fig. 3. This verifies the proper epigrowth of $\sim 5 \mathrm{~nm}$ thick $\mathrm{In}_{0.35} \mathrm{Ga}_{0.65} \mathrm{~N} / \mathrm{GaN}$ quantum structures. In forward bias, these InGaN/GaN devices exhibit strong electroluminescence (EL). Figure 2 also shows the EL spectrum when the diode is driven with $10 \mathrm{~mA}$ of current, along with a micrograph of the blue light emitted from the device. The optical power coupled from one side is $>0.35 \mathrm{~mW}$ at a current injection level of $20 \mathrm{~mA}$. EL spectrum peaks at $437 \mathrm{~nm}$ (redshifted with respect to PL) with a FWHM of $25 \mathrm{~nm}$.

We measure the optical absorption spectra using our photocurrent measurement setup including a monochromator, a xenon lamp, a lock-in amplifier, a power meter, and a dc power supply for the application of various reverse bias voltages. Figure 1 shows the optical absorption spectra from 400 to $460 \mathrm{~nm}$ parametrized with the applied reverse biases from 0 to $-6 \mathrm{~V}$ (with the dark current levels in the range of few nanoamperes). Here we observe that the optical absorption edge blueshifts with the increasing applied reverse field, unlike in the case of the conventional QCSE. This blueshift is a direct experimental evidence of the polarization fields seen in the wells in the opposite direction of the applied reverse field (thus, in the opposite direction of the built-in field). Therefore, the well polarization field lies in the opposite direction of the epi-growth and extends from the top epitaxial layer ( $p$ side) toward the bottom epitaxial layer ( $n$ side), verifying the expected direction of the polarization in the InGaN wells on polar $c$ plane. ${ }^{9}$ Also, in Fig. 1, we observe an inflection point at $418 \mathrm{~nm}$ on the absorption curves of our InGaN/GaN quantum structures under different biases, which is in agreement with the work of Friel et al. on GaN/AlGaN quantum structures. ${ }^{10}$ For reverse biases close to $-6 \mathrm{~V}$ and beyond, the barriers that have polarization in the same direction as the applied reverse field turn into considerably thin potential barriers, exhibiting transition from the quantum confined case to the bulk case, also observed in Fig. 


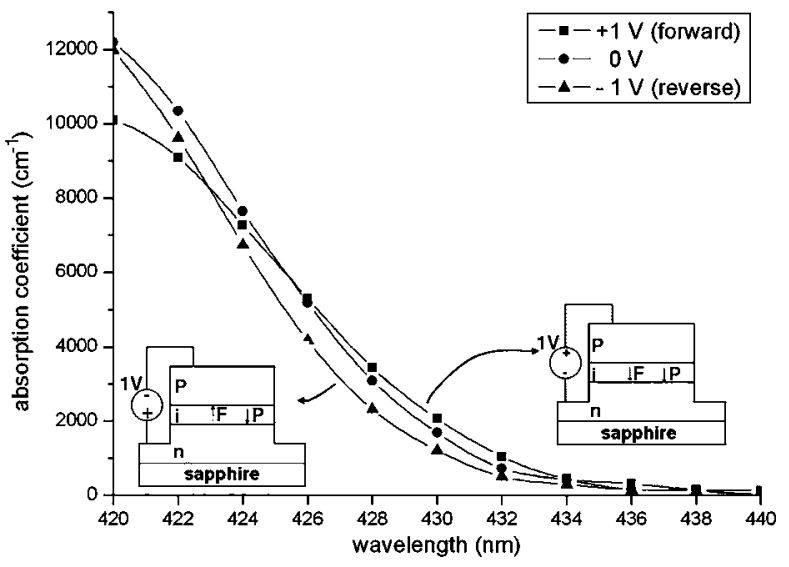

FIG. 4. Optical absorption curves measured under $1 \mathrm{~V}$ (forward bias), $0 \mathrm{~V}$ (no bias), and $-1 \mathrm{~V}$ (reverse bias) at room temperature.

1. From the compensation of the polarization field with the applied field, we experimentally predict the intrinsic polarization field in the wells to be $120 \mathrm{~V} / \mu \mathrm{m}$, which is comparable to the theoretically expected $300 \mathrm{~V} / \mu \mathrm{m}$.

Figure 4 shows the optical absorption spectrum of our InGaN/GaN quantum zigzag structures when a forward bias of $1 \mathrm{~V}$ is applied, along with the spectra under a reverse bias of $-1 \mathrm{~V}$ and no bias for comparison. Here we observe that the absorption spectra differ for different directions of bias application (despite comparable levels of leakage current). The absorption spectrum appears more to redshift due to the traditional QCSE when forward biased (i.e., when the electric field $F$ is applied in the direction of the well polarization $P)$ and to blueshift due to the reversed QCSE when reverse biased (i.e., when $F$ is applied in the opposite direction of $P$ ).

Figure 5 shows the electroabsorption coefficient change (with respect to the no-bias case) with the largest change of $6000 \mathrm{~cm}^{-1}$ for a $6 \mathrm{~V}$ swing (corresponding to $50 \mathrm{~cm}^{-1}$ absorption coefficient change for $1 \mathrm{~V} / \mu \mathrm{m}$ field change) at $424 \mathrm{~nm}$. Here the blue region between 420 and $430 \mathrm{~nm}$ is a very good operating wavelength range for such a modulator with large absorption change and low background absorption (as low as $767 \mathrm{~cm}^{-1}$ ). In Fig. 5, we also show the optical transmission spectra modulated with the reverse bias in the inset. The transmission and absorption spectra lead to the same observations of the blueshift and inflection point at $418 \mathrm{~nm}$. Here, in the surface-normal configuration, the short optical interaction length of $50 \mathrm{~nm}$ (along with 17\% stray light not passing through the mesa) renders a low transmission modulation of $3.05 \%$ at $424 \mathrm{~nm}$ for $5 \mathrm{~V}$ bias, as also expected by the absorption spectra.

For electroabsorption, we observe a maximum optical absorption change of $67 \mathrm{~cm}^{-1} / 1 \mathrm{~V} / \mu \mathrm{m}$ field swing between the reserve biases 0 and $-2 \mathrm{~V}$. This electroabsorption effect is stronger than those previously observed in bulk $\mathrm{GaN}$ based materials $^{11}$ and in InGaN/GaN quantum structures of blue laser diodes. ${ }^{12}$ This implies that a $100 \mu \mathrm{m}$ long waveguide modulator with a reasonable overlap integral of the waveguide mode with our $\mathrm{InGaN} / \mathrm{GaN}$ structures (e.g., $\Gamma \sim 0.1$ ) is expected to achieve a $10 \mathrm{~dB}$ contrast ratio for a $2 \mathrm{~V} / \mu \mathrm{m}$ field swing. This further means that the electroabsorption performance of these InGaN/GaN quantum zigzag structures in the blue is comparable to that of the InGaAsP/InP quantum well structures in the near IR (e.g., $c$ band), ${ }^{13,14}$ also commercially used in communications. Furthermore, be-

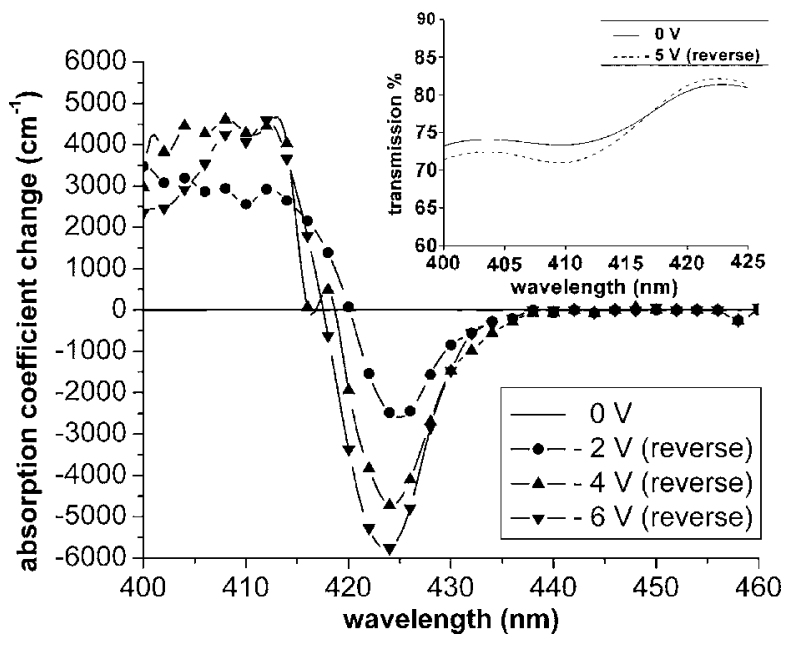

FIG. 5. Optical absorption coefficient change with respect to $0 \mathrm{~V}$, along with transmission spectra at 0 and $-5 \mathrm{~V}$ in the inset.

cause of the low parasitic in-series resistance $(<100 \Omega)$ and low capacitance $\left(<100 \mathrm{fF}\right.$ for a $3 \times 100 \mu \mathrm{m}^{2}$ lateral device area), blue InGaN/GaN quantum electroabsorption modulators hold promise for $>10 \mathrm{GHz}$ optical clock generation and injection directly into Si CMOS chips in the blue. Additionally, the electroluminescence of these $\mathrm{InGaN} / \mathrm{GaN}$ quantum devices in the blue range makes it possible to consider onchip integration of blue light source and modulator for a compact optical clocking scheme.

In conclusion, we presented blue $\mathrm{InGaN} / \mathrm{GaN}$ based quantum electroabsorption modulators that find application in blue optical clock injection directly into $\mathrm{Si}$ CMOS, unlike in the infrared. Using our InGaN/GaN quantum zigzag structures, we demonstrated strong electroabsorption behavior with a blueshift due to the reversed quantum confined Stark effect.

This work is supported by EU MOON 021391, EU NOE PHOREMOST 511616, Turkish Academy of Sciences TUBA GEBIP, and TUBITAK EEEAG 104E114, 106E020, 105E065, and 105E066.

${ }^{1}$ D. A. B. Miller and H. M. Özaktas, ARTE CEMENTO 41, 42 (1997).

${ }^{2}$ J. W. Goodman, F. I. Leonberger, and S. Y. Kung, Proc. IEEE 72, 850 (1984).

${ }^{3}$ D. A. B. Miller, Int. J. Eng. Sci. 11, 155 (1997).

${ }^{4}$ G. Li, D. Huang, E. Yuceturk, P. J. Marchand, S. C. Esener, V. H. Ozguz, and Y. Liu, Appl. Opt. 41, 348 (2002).

${ }^{5}$ T. K. Woodward and A. V. Krishnamoorthy, Electron. Lett. 34, 1252 (1998).

${ }^{6}$ A. Bhatnagar, S. Latif, C. Debaes, and D. A. B. Miller, J. Lightwave Technol. 22, 2213 (2004).

${ }^{7}$ D. A. B. Miller, D. S. Chemla, T. C. Damen, A. C. Gossard, W. Wiegmann, T. H. Wood, and C. A. Burrus, Phys. Rev. B 32, 1043 (1985).

${ }^{8}$ V. Fiorentini, F. Bernardini, F. D. Sala, A. Di Carlo, and P. Lugli, Phys. Rev. B 60, 8849 (1999).

${ }^{9}$ T. Takeuchi, C. Wetzel, S. Yamaguchi, H. Sakai, H. Amano, I. Akasaki, Y. Kaneko, S. Nakagawa, Y. Yamaoka, and N. Yamada, Appl. Phys. Lett. 73, 1691 (1998).

${ }^{10}$ I. Friel, C. Thomidis, and T. D. Moustakas, J. Appl. Phys. 97, 123515 (2005).

${ }^{11}$ A. E. Oberhofer, J. F. Muth, M. A. L. Johnson, Z. Y. Chen, and E. F. Fleet, Appl. Phys. Lett. 83, 2748 (2003).

${ }^{12}$ M. Kneissl, T. L. Paoli, P. Kiesel, D. W. Treat, M. Teepe, N. Miyashita, and N. M. Johnson, Appl. Phys. Lett. 80, 3283 (2002).

${ }^{13}$ N. C. Helman, J. E. Roth, D. P. Bour, H. Altug, and D. A. B. Miller, IEEE J. Sel. Top. Quantum Electron. 11, 338 (2005).

${ }^{14}$ H. V. Demir, V. A. Sabnis, O. Fidaner, J. S. Harris, Jr., D. A. B. Miller, and J.-F. Zheng, IEEE J. Quantum Electron. 11, 86 (2005). 